\title{
Ultrasonic bandgaps in 3D-printed periodic ceramic microlattices
}

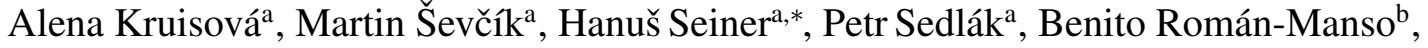 \\ Pilar Miranzo ${ }^{\mathrm{c}}$, Manuel Belmonte ${ }^{\mathrm{c}}$, Michal Landa ${ }^{\mathrm{a}}$ \\ ${ }^{a}$ Institute of Thermomechanics, Czech Academy of Sciences, Dolejškova 5, 18200 Prague, Czech Republic. \\ ${ }^{b}$ School of Engineering and Applied Sciences, Harvard University, \\ 52 Oxford St, 02318 Cambridge MA, USA \\ ${ }^{c}$ Institute of Ceramics and Glass (ICV-CSIC), Campus de Cantoblanco, Kelsen 5, 28049 Madrid, Spain
}

\begin{abstract}
The transmission of longitudinal ultrasonic waves through periodic ceramic microlattices fabricated by Robocasting was measured in the $2-12 \mathrm{MHz}$ frequency range. It was observed that these structures (scaffolds of tetragonal and hexagonal spatial arrangements with periodicity at length-scales of $\sim 100 \mu \mathrm{m}$ ) exhibit well-detectable acoustic band structures with bandgaps. The locations of these gaps were shown to be in close agreement with the predictions of numerical models, especially for the tetragonal scaffolds. For the hexagonal scaffolds, a mixing between longitudinal and shear polarizations of the propagation modes was observed in the model, which blurred the matching of the calculated band structures with the experimentally measured bandgaps.

Keywords: Phononic crystals; Ceramics; Additive manufacturing; Bandgaps; Wave propagation; Finite elements method.
\end{abstract}

Robocasting $[1,2,3]$ is an additive manufacturing technique that enables fabrication of micro-architectured three-dimensional (3D) scaffolds consisting of ceramic micro-rods in periodic spatial arrangements. These scaffolds are typically developed for particular applications such as bone tissue engineering [4, 5] or advanced liquid filtering [6]; however, the high acoustic performance of dense ceramic materials in combination with the periodicity of the micro-architecture of the scaffold predetermines the robocast structures also for applications in acoustics.

\footnotetext{
*Corresponding author, hseiner@it.cas.cz
} 
As previously shown by the current authors [7], tetragonal robocast scaffolds exhibit extremely strong elastic anisotropy, accompanied by equivalently anisotropic effects in the acoustic wave propagation. In addition, due to the periodicity, a pronounced frequency-dependence of the acoustic wave propagation can be expected, as some specific frequencies of the propagating waves may induce internal resonances of the structure, which prevents the acoustic energy from being transmitted through the scaffold. Hence, the existence of the so-called frequency bandgaps (BGs) typical for acoustic metamaterials $[8,9,10]$ or phononic crystals $[11,12,13,14]$ can be theoretically expected for the scaffolds. Indeed, the appearance of these gaps in tetragonal robocast structures was recently predicted by finite elements calculations $[7,15]$.

In this paper, we give experimental evidence of the existence of acoustic BGs for silicon carbide $(\mathrm{SiC})$ scaffolds of various micro-architectures and symmetry classes. We show that the BG structure can be reliably predicted by finite elements calculations, especially for scaffolds with highly symmetric (tetragonal) spatial arrangements. The main aim of this paper is, however, to show experimentally that the acoustic energy transmission through a robocast scaffold is strongly dependent on the micro-architecture, which opens the possibility of tuning this architecture in order to achieve the desired acoustic filtering properties. As shown by Liu et al. [16, 17] and Kuang et al. [18], the structure and width of acoustic BGs of a porous material with geometrically ordered pores are strongly dependent on the shape of the pores and their volume fraction. In principle, the Robocasting method enables fine tailoring of both the porosity and the geometry of pores.

There have been several experimental demonstrations of phononic crystals reported in the literature so far $[19,20,21,22]$, typically exhibiting bandgaps in a $100 \mathrm{~Hz}-100 \mathrm{kHz}$ frequency range, as the spatial periodicity in these structures appeared at the millimeter (or even longer) length-scales. In contrast, the robocast scaffolds reported in this paper have the periodicity at approximately ten to hundred times smaller characteristic lengthscales; this, together with the high acoustic impedance of fully dense ceramics, enables the robocast materials to exhibit BGs and other metamaterial-like features at ultrasonic frequencies $\sim 5-10 \mathrm{MHz}$, which has rarely been reported in the literature yet [23]. 


\section{Experimental}

\subsection{Materials}

The examined SiC micro-scaffolds were fabricated by Robocasting using a similar additive manufacturing process as described in [3,24]. In brief, an aqueous colloidal paste (containing attrition milled $\mathrm{SiC}$ powders with 7 wt. $\% \mathrm{Al}_{2} \mathrm{O}_{3}$ and $\mathrm{Y}_{2} \mathrm{O}_{3}$ sintering additives plus organic additives) was printed through a robotically controlled nozzle into an oil bath, so that a regular architecture was obtained layer-by-layer, consisting of a linear array of parallel filaments in each layer. By different mutual rotations between these layers and different spacings between the rods, four different micro-architectures were obtained, according to CAD computer designs shown in Fig.1(a). Two samples were of tetragonal symmetry (LS and SS), differing only in the in-plane spacings between the rods; similarly, two samples were of hexagonal arrangements ( $\mathrm{LH}$ and $\mathrm{SH})$. Hereafter, we will use the acronyms (LS, SS, LH, and SH) to denote the samples.

Each structure was then slowly dried at room temperature and subsequently heat treated at $600{ }^{\circ} \mathrm{C}$ to burn out the organic additives. As a final step, the 3D structures were densified by pressureless Spark Plasma Sintering (SPS) at $1700{ }^{\circ} \mathrm{C}$ in Ar. As shown in [24], these sintering conditions lead to full densification of the ceramic materials. Scanning Electron Microscopy (SEM) images and optical micrographs of the final sintered micro-lattices are shown in Figs. 1(b) and 1(c), respectively. From the four prepared micro-architectures, samples for ultrasonic measurement were prepared by cutting out the non-periodic parts of the scaffolds (Fig. 1(d)).

\subsection{Experimental Methodology}

The acoustic properties of the samples were studied using a through-transmission method (see Fig.2 for an outline). Each sample was contacted by a CrystalBond wax onto two $0.5 \mathrm{~mm}$ thin glass plates to ensure flat co-planar faces for contacting of ultrasonic probes.

Then, narrow-band longitudinal ultrasonic pulses were sent through the sample, each consisting of ten harmonic periods at given frequency inside of an amplitude-envelope ('input signal' curve in Fig.2). Glass plates were always glued onto two lateral faces of 
(a)

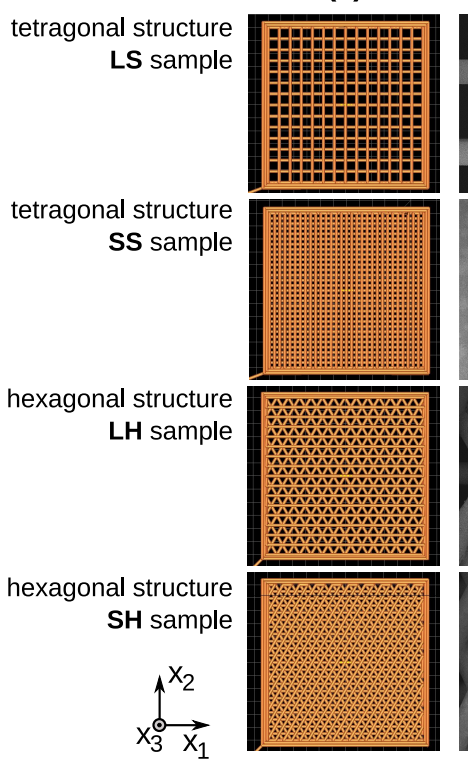

(b) $500 \mu \mathrm{m}$

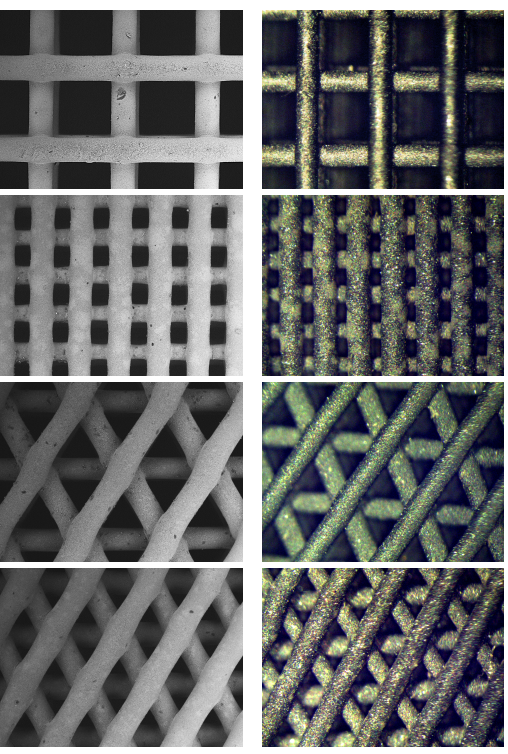

(d)

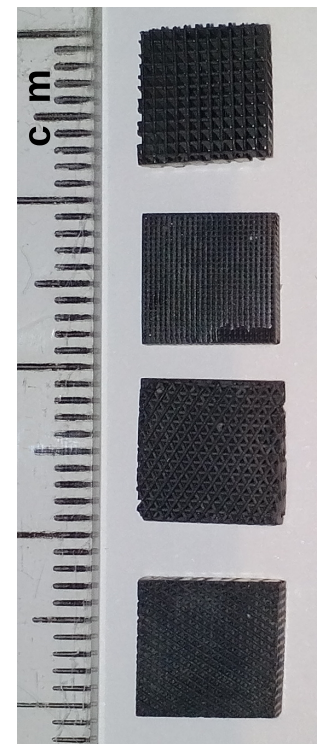

Figure 1: The four different micro-architectures used in the experiments: (a) initial computer CAD designs; (b) SEM images of the resulting sintered architectures; (c) optical micrographs of these architectures; (d) samples for ultrasonic measurements cut from the central parts of the robocast structures.

the sample and oriented such that they enabled measurements of waves propagating along one of the orientations of the rods. According to the coordinate system outlined in Fig.1, the measurements were performed in the $x_{1}$ direction for all samples.

For the generation of the input signals and the acquisition of the transmitted signals, a National Instruments NI-PXI card system was used, including a functional generation card 5422 (16 bits/40 MHz) employed for burst signal generation, and an acquisition card 5122 (14 bits/100 MHz) for signal recording. The generated as well as the recorded sig-

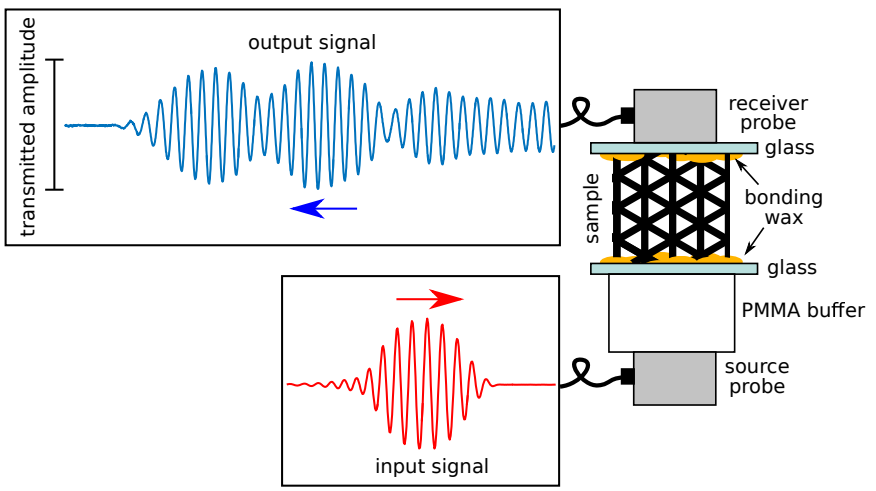

Figure 2: Outline of the experimental set-up. 


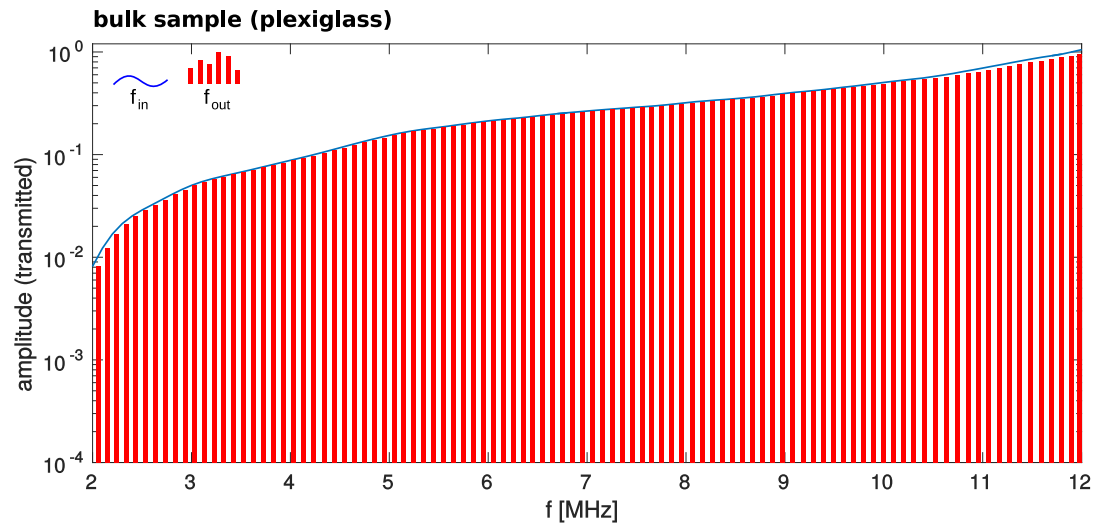

Figure 3: Results of a preliminary reference measurement for a bulk plexiglass sample. The solid (blue) line is a plot the transmitted amplitude with respect to the set input frequency $f_{\text {in }}$ of the transmitter probe, the vertical (red) bars represent the plot of the same amplitude with respect to the dominant frequency of the output signal $f_{\text {out }}$.

nals were amplified by a high power ultrasonic system Ritec RAM 5000. This system was equipped by a gated amplifier (nominal frequency range $50 \mathrm{kHz}-22 \mathrm{MHz}$, output RMS power $5 \mathrm{~kW}$ at $50 \mathrm{ohms} / 2.5 \mathrm{MHz}$ ) and a broadband receiver (frequency band $40 \mathrm{kHz}$ $80 \mathrm{MHz} / 72 \mathrm{~dB}$ gain).

A $\mathrm{LiNbO}_{3}$ single crystal (central frequency $20 \mathrm{MHz}$ ) glued on a $10 \mathrm{~mm}$ Homosil substrate was used as an emitting transducer. A commercial delayed ultrasonic probe Panametric V206 (0.12”, $20 \mathrm{MHz}$ ) was employed as a receiver. Propylenglycol was used for acoustic coupling.

Input burst pulses with nominal frequencies $\left(f_{\text {in }}\right)$ between $2 \mathrm{MHz}$ and $12 \mathrm{MHz}$ were used; this frequency range was scanned with a $0.1 \mathrm{MHz}$ step.

While the input signal was a compact burst wave-packet, the transmitted signal was much more general (as, for example, the 'output signal' curve in Fig.2). For each nominal frequency of the input signal, two basic parameters of the output signal were determined: 1) the maximal transmitted amplitude, and 2$)$ the dominant frequency $\left(f_{\text {out }}\right)$ determined by Fast Fourier Transform (FFT) of the output signal. It was checked that for a reference sample (a $10 \mathrm{~mm}$ thick bulk plexiglass) the transmitted amplitude was monotonously increasing with frequency for the whole used frequency range, and that

$$
f_{\text {in }}=f_{\text {out }}
$$

in the given frequency range. This result is visualized in Fig. 3 as a plot of the transmitted 
amplitude with respect to the input frequency $f_{\text {in }}$ (solid line) and the output frequency $f_{\text {out }}$ (vertical bars). The amplitude is normalized with respect to its maximum value (reached at $12 \mathrm{MHz}$ ). It is seen that the amplitude decreased below $3 \mathrm{MHz}$ due to low sensitivity of the detection probe to such low frequencies; in the rest of the used frequency range, the amplitude was increasing due to approaching the vicinity of the resonant frequencies of both emitting and receiving probes. This reference measurement confirmed that any localized sharp decreases in the transmitted amplitude obtained for the robocast samples must follow from the interactions of the propagating waves with the micro-architecture. In the rest of the paper, we will use the same representation of the acoustic energy transmission through the robocast samples as the one used in Fig. 3 for the bulk plexiglass sample, i.e. the plots of the transmitted amplitude with respect to the input and output frequencies.

\section{FEM calculations}

In addition to the experiment, the wave propagation in the examined scaffolds was studied by numerical modelling, in particular by the finite elements method (FEM). The calculations were performed on representative unit cells of the scaffolds shown in Fig.4. For both tetragonal and hexagonal scaffolds these unit cells are representative building blocks that repeat periodically along the three spatial directions $\left(x_{1}, x_{2}\right.$ and $\left.x_{3}\right)$. However, while for the tetragonal structures this building block is directly equivalent to the smallest (irreducible) unit cell of the scaffold, for the hexagonal structures the cell used for the calculation is composed of two mirror-reflected irreducible unit cells, each of them periodically repeating in the structure along the directions $x_{2}= \pm \sqrt{3} x_{1}$.

The unit cells were meshed by tetrahedral Lagrangian elements, and the corresponding wave-propagation problem was solved using COMSOL Multiphysics computational environment [25]. The dimensions of the unit cells, i.e. the out-of-plane spacing of the rods $h$, the in-plane spacing of the rods $l$, and the rod thickness $d$, were obtained from optical micrographs of the scaffolds (both axial views and cross-sectional cuts); the results are listed in Table 1. A relatively large scatter (up to $\pm 20 \mu \mathrm{m}$ ) was observed in the micrographs for all parameters. Hence, the results of the FEM calculations must be understood just as idealized approximations of the behaviors of the real scaffolds. The material pa- 

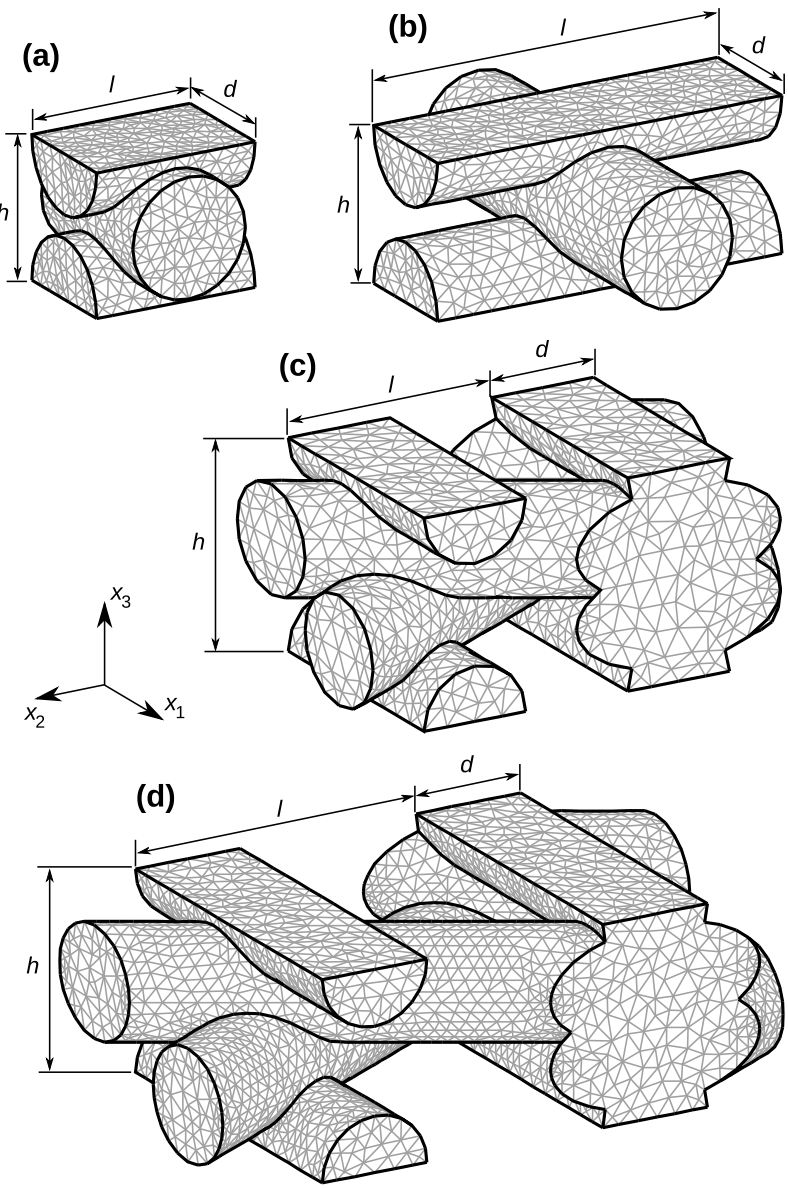

Figure 4: Geometries and FEM meshes used for calculations of the band structure of the studied scaffolds: (a) SS structure; (b) LS structure; (c) SH structure; (d) LH structure.

rameters of robocast $\mathrm{SiC}$ for the calculations were taken from [7] as follows: the density $\rho=3.34$ g.cm ${ }^{-3}$, Young's modulus $E=305 \mathrm{GPa}$, and Poisson's ratio $\nu=0.19$.

As usual for wave propagation in periodic media with spatial periodicity described by a translational vector a $[19,26,27]$, the displacement field of the sought solution was assumed in form of a planar wave of frequency $\omega$

$$
\mathbf{u}(\mathbf{x}, t)=\mathbf{U}_{\mathrm{B}}(\mathbf{x}, \mathbf{k}) \exp [i(\mathbf{k} \cdot \mathbf{x}-\omega t)]
$$

where

$$
\mathbf{U}_{\mathrm{B}}(\mathbf{x}, \mathbf{k})=\mathbf{U}_{\mathrm{B}}(\mathbf{x}+\mathbf{a}, \mathbf{k})
$$

is the Bloch waveform $[28,29]$ having the same spatial periodicity as the scaffold, and $\mathrm{k}$ is the wave vector, that can be always mapped into the first irreducible Brillouin zone. 


\begin{tabular}{lccc}
\hline \hline Sample & $l[\mu \mathrm{m}]$ & $d[\mu \mathrm{m}]$ & $h[\mu \mathrm{m}]$ \\
\hline LS & 650 & 210 & 275 \\
SS & 308 & 219 & 261 \\
LH & 576 & 215 & 385 \\
SH & 420 & 210 & 405 \\
\hline \hline
\end{tabular}

Table 1: Geometric parameters of the calculation domains for FEM calculations; see Fig.4 for the meaning of individual parameters.

In particular, planar waves propagating along the symmetry direction $x_{1}$ were assumed in all cases; in this direction, the spatial period of the scaffolds was $a=l$ for the tetragonal structures and $a=2 l / \sqrt{3}$ for the hexagonal structures. The calculations were done for sets of wave numbers $k=|\mathbf{k}| \in[0, \pi / a]$ for the tetragonal structures, and $k=|\mathbf{k}| \in$ $[0,2 \pi / a]$ for the hexagonal ones.

The Bloch wave solution was determined by calculating eigenfrequencies and eigenmodes of elastic vibrations of the unit cell with the complex phase-shift boundary condition

$$
\mathbf{u}\left(x_{1}=0, x_{2}, x_{3}, t\right)=\mathbf{u}\left(x_{1}=a, x_{2}, x_{3}, t\right) \exp (i k a)
$$

in direction $x_{1}$ and periodic boundary conditions in the $x_{2}$ and $x_{3}$ directions. For the tetragonal scaffolds, this calculation gave directly the full band structure for the wave propagation in direction $x_{1}$. For the hexagonal scaffolds, the solutions were duplicate due to the fact that the used computational unit cell consisted of two irreducible unit cells of the structure; i.e. for each $k \in[0,2 \pi / a]$, also solutions for $\widetilde{k}=(2 \pi / a)-k$ were obtained. The separation of these two sets was done by checking the phase shifts between corresponding points at planes $x_{1}=0$ and $x_{1}=a / 2$.

For each solution, the displacement amplitudes $u_{1}$ (i.e. along the propagation direction), $u_{2}$ and $u_{3}$ at the faces perpendicular to $x_{1}$ were compared, which enabled identification of waves with dominantly longitudinal polarizations. For a more detailed analysis of the individual modal shapes, visualizations of the corresponding solutions on supercells consisting of four unit cells repeating along the propagation direction were constructed from the solutions on single cells. 


\begin{tabular}{lllll}
\hline \hline Sample & LS & SS & LH & SH \\
\hline $\begin{array}{l}\text { 1st cut-off freq. } \\
\text { 2nd cut-off freq. }\end{array}$ & 3.3 & 9.5 & 3.8 & 6.1 \\
\hline $\begin{array}{l}\text { 1st BG location } \\
\text { experimental }\end{array}$ & $3.5-4.9$ & $9.6-11.5$ & $4.3-5.4$ & $6.1-7.8$ \\
calculated & $3.2-4.5$ & from 10.1 & $3.8-5.4$ & $5.7-7.9$ \\
\hline $\begin{array}{l}\text { 2nd BG location } \\
\text { experimental }\end{array}$ & $7.7-9.1$ & & & \\
calculated & $6.9-7.8$ & & $6.3-8.5$ & from 11.0 \\
\hline $\begin{array}{l}\text { 3rd BG location } \\
\text { experimental }\end{array}$ & from 11.1 & & $6.2-7.9$ & $9.8-10.6$ \\
calculated & from 11.6 & & $9.6-11.5$ & \\
\hline \hline
\end{tabular}

Table 2: Cut-off $(-60 \mathrm{~dB})$ frequencies and BGs of the examined samples. All values are in MHz (experimental data with accuracy $\pm 0.1 \mathrm{MHz}$ ). 
Figure 5: Experimental results for the tetragonal scaffolds LS (upper plot) and SS (lower plot). The solid line is the plot of the transmitted amplitude with respect to the input frequency; the vertical bars are the plot with respect to the dominant frequency of the output signal. For the $f_{\text {out }}$ plot, approximate locations of the BGs are outlined by the dashed lines; broken arrows denote the locations of the drops of the normalized amplitude beyond $10^{-3}$ (i.e. $-60 \mathrm{~dB}$ ).

For the SS sample, a shallow local minimum is observed at about $4.5 \mathrm{MHz}$, but there is no real gap in the transmitted frequencies related to this minimum. A strong decrease in the transmitted amplitude is then seen at above $9 \mathrm{MHz}$, reaching a $-60 \mathrm{MHz}$ cut-off frequency at 9.5 MHz. A sharp BG for the SS structure is localized between 9.8 and 10.6 MHz. Let us mention that the decreases of the amplitudes around the BGs are gradual rather than abrupt for both samples, which is probably due to the irregularities of the real structure mentioned in Section 3, that leads to blurring of the BG edges.

The observed band structure for the LS and SS samples can be easily interpreted based on the results of the FEM modeling shown in Fig. 6. For the LS sample, the FEM model predicts that there are three longitudinal modes appearing in the studied frequency range. Two of them with positive group velocity $(\partial \omega / \partial k>0$, modes denoted by capital letters 
$\mathrm{C}-\mathrm{C}^{\prime}$ and D-D $\mathrm{D}^{\prime}$ in Fig.6) and one with negative group velocity $(\partial \omega / \partial k<0$, mode $\mathrm{G}-\mathrm{G}^{\prime}$ ). Fig. 7 shows the modal shapes of chosen modes for two different values of the $k$-vector: in the middle between the center and the edge of the 1st Brillouin zone ( $k=a / 2 \pi)$ and at the edge of the 1st Brillouin zone $(k=a / \pi)$, where the size of the unit cell corresponds to one half of the wavelength. It is observed that the geometry of the modes for these two values of $k$ is essentially the same, up to the change in the wavelength.

From these mode visualizations, it is clearly seen that these three modes represent purely longitudinal straining of the scaffold, while all other modes have dominantly shear polarizations related to bending of the rods oriented along the propagation direction. The high symmetry class of the scaffold enabled clear distinguishing between the shear and longitudinal modes, according to the symmetry components of the structure. In summary, there is a good quantitative agreement between the gaps separating the modes $\mathrm{C}-\mathrm{C}^{\prime}$, $\mathrm{D}-\mathrm{D}^{\prime}$ and $\mathrm{G}-\mathrm{G}^{\prime}$ and the experimentally observed BGs.

For the SS sample, the FEM calculation predicts only one BG within the measured frequency range, starting above the edge of the first longitudinal mode and denoted by a thick line in the lower plot of Fig.6. This mode is geometrically equivalent to the $\mathrm{C}-\mathrm{C}^{\prime}$ mode in the LS structure. The quantitative agreement between the FEM prediction of the location of this BG and the experimental value is also satisfactory, especially when taking into account that for higher frequencies (shorter wavelengths) the local imperfections in the geometry of the structure, i.e. deviations of the real geometry of the scaffold from the idealized geometry of the FEM unit cell, may have a more pronounced effect. The FEM model also confirms that there should be no $\mathrm{BG}$ around $4 \mathrm{MHz}$, i.e. that the first local minimum on the amplitude curve of the SS sample is artificial, probably corresponding to the thickness resonance of the glass plates used for contacting of the sample.

\subsection{Hexagonal structures}

For the hexagonal LH and SH samples, the experimental results are shown in Fig.8. Localized decreases in the amplitude followed by broad BGs can also be observed for these structures. The frequency ranges of these gaps are summarized in the third and the fourth columns of Table 2. For the LH sample, a sharp BG is observed between 6.3 and 

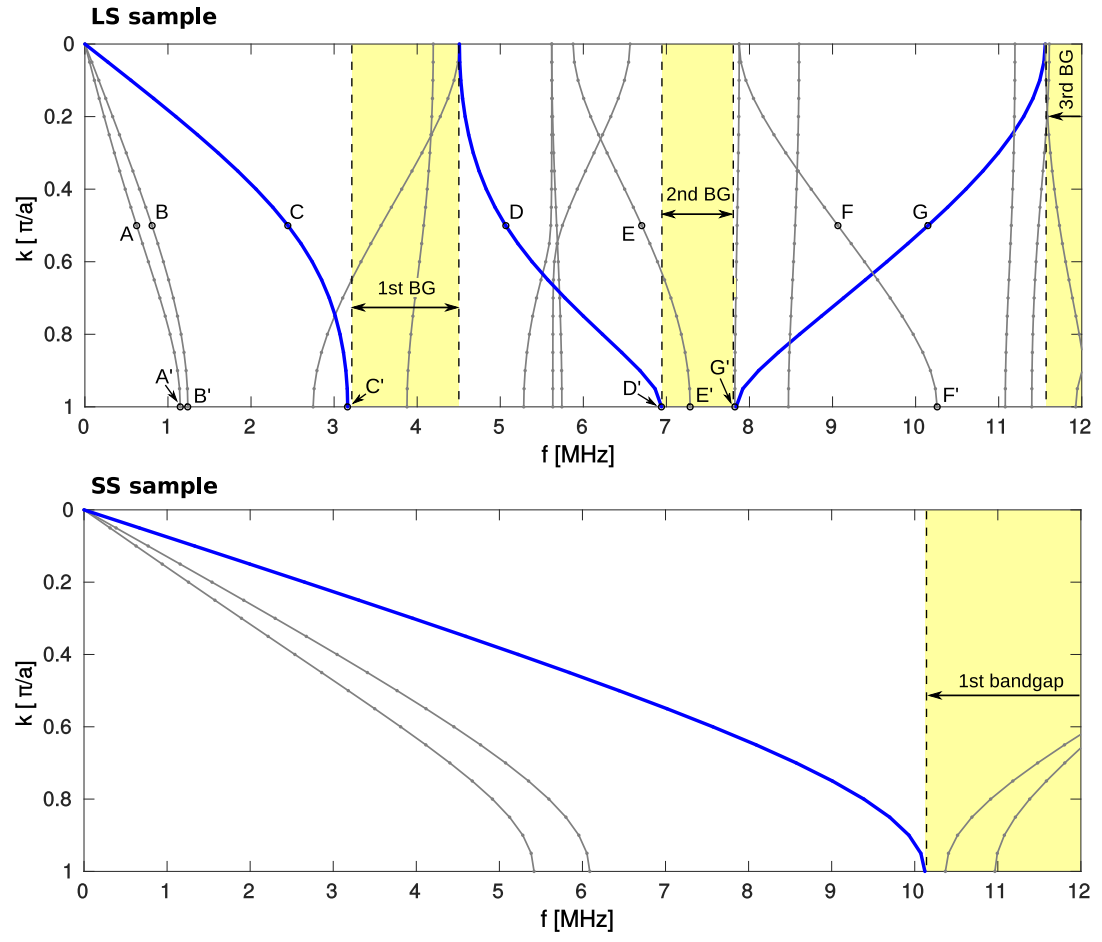

Figure 6: Band structure of the tetragonal scaffolds LS (upper plot) and SS (lower plot) calculated by FEM. The thick (blue) lines denote the modes with dominant longitudinal component; the shaded (yellow) areas denote the gaps between these modes. In the upper plot, the capital letters $A, \ldots, G$ and $A^{\prime}, \ldots, G^{\prime}$ labeling the individual modes refer to Fig.7.

8.5 MHz, while for the SH sample the BGs are detected between 6.1 and $7.8 \mathrm{MHz}$ and above $11.0 \mathrm{MHz}$. For the LH sample, two less pronounced minima of the transmitted amplitude can be also seen. The first appears above the first $-60 \mathrm{~dB}$ cut-off frequency (3.8 MHz) and corresponds to the $f_{\text {out }}$ frequency range denoted as '1st BG' in Fig.8. The second minimum ('3rd BG') is very shallow, located approximately between 9.6 and 11.5 MHz. It is not clear from the experimental results whether the latter minimum can be assigned to some real BG.

The interpretation of the observed band structure for the hexagonal samples by FEM calculations (Fig.9) is much less straightforward than for the case of the tetragonal structures. Most importantly, the propagation along the $x_{1}$ direction in the hexagonal structure does not correspond to a single direction on the 1st Brillouin zone, but to a path from the center of the zone $(\Gamma)$ to its corner (point $K$ ) and then to its edge (point M, see the sketch in Fig.9), where the notation $\Gamma, \mathrm{M}$ and $\mathrm{K}$ is adopted from phonon mechanics [29]. Due to this complex path, the $\omega(k)$ curves for most of the propagation modes are non-monotonous, 
Figure 7: Visualizations of the selected propagation modes (see the labels in Fig. 6) for the LS structure. Modal shapes $\mathrm{A}, \ldots, \mathrm{G}$ correspond to $k=\pi / 2 a$, modal shapes $\mathrm{A}^{\prime}, \ldots, \mathrm{G}^{\prime}$ to $k=\pi / a$. The color-scale represents the instantaneous displacement amplitude for fixed phase angle $\omega t$.

exhibiting local stationary points $(\partial \omega / \partial k=0)$ for various values of $k \leq 2 \pi / a$.

The visualizations of the selected propagation modes (see the labels in Fig. 9) for the LH structure are shown in Fig. 10 for $k=\pi / 2 a, k=\pi / a$ and $k=2 \pi / a$. Among the three lowest (acoustic) modes, two shear modes $\left(\mathrm{A}-\mathrm{A}^{\prime}-\mathrm{A}^{\prime \prime}, \mathrm{B}-\mathrm{B}^{\prime}-\mathrm{B}^{\prime \prime}\right)$ and one longitudinal mode $\left(\mathrm{C}-\mathrm{C}^{\prime}-\mathrm{C}^{\prime \prime}\right)$ can be easily distinguished based on the modal shapes, although the latter intersects already with a higher-order shear mode $\mathrm{D}-\mathrm{D}^{\prime}-\mathrm{D}^{\prime \prime}$. The longitudinal mode has a stationary point at approximately $k=0.6(2 \pi / a)$, i.e. neither in the corner of the zone (but close to it), nor at its edge. The fact that the stationary point does not appear exactly at the corner of the 1st Brillouin zone follows probably from the mode conversion (from longitudinal to shear) by Bragg scattering at the K-point. This stationary point, however, corresponds well to the experimentally observed first cut-off frequency and the start of the first BG. The polarizations of the modes appearing above this point are dominantly shear, except of the mode $E-E^{\prime}-E^{\prime \prime}$, which is a propagating longitudinal mode 

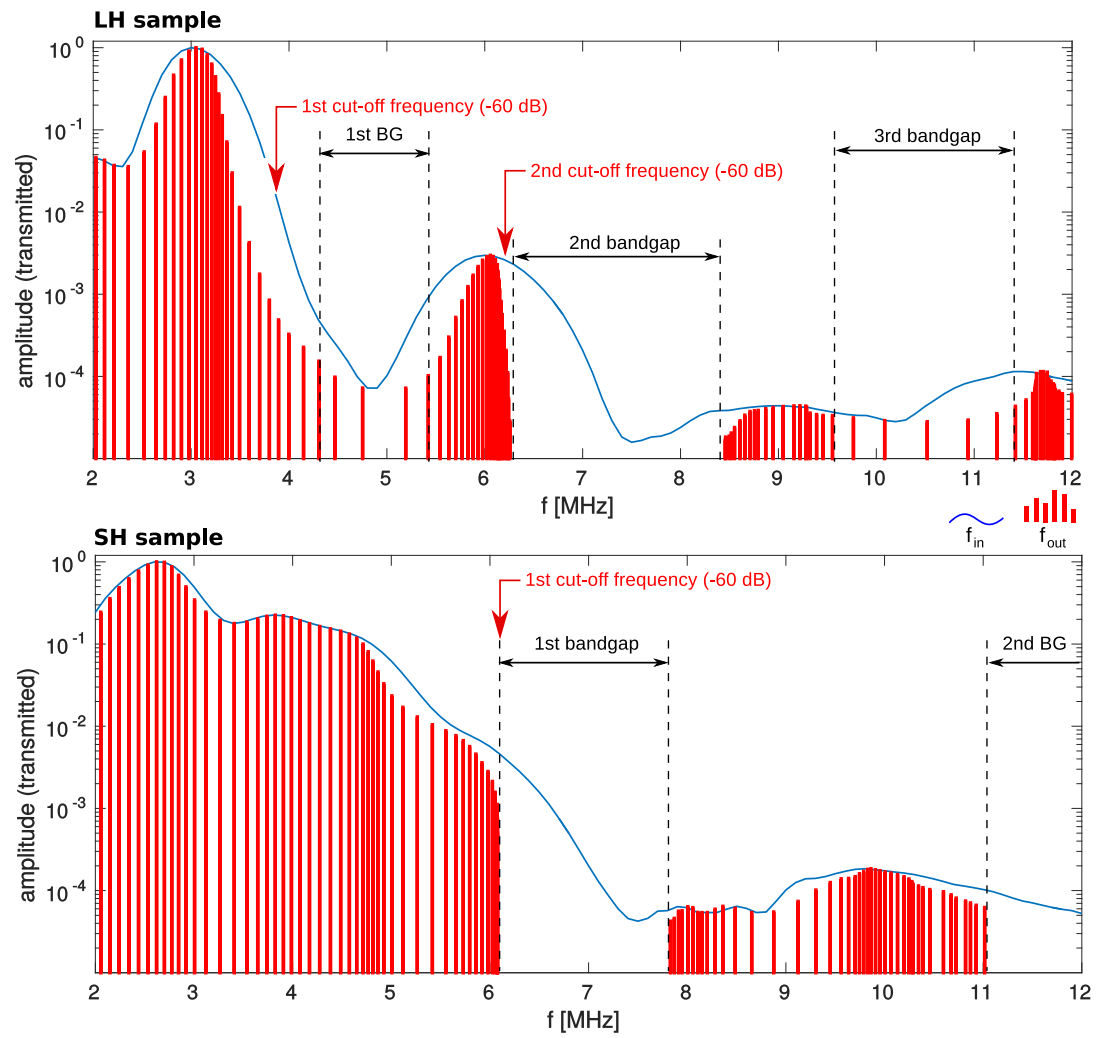

Figure 8: Experimental results for the hexagonal scaffolds LH (upper plot) and SH (lower plot); see Fig.5 for a detailed legend.

between points $\Gamma$ and $\mathrm{K}$, but turns into a non-propagating mode $(\partial \omega / \partial k=0)$ between $\mathrm{K}$ and M. The upper edge frequency of this mode corresponds well to the start of the second experimentally observed BG in the LH structure. At higher frequencies, nevertheless, any clear distinguishing between longitudinal and shear modes becomes impossible. Unlike for the tetragonal scaffolds, the hexagonal scaffolds do not impose any particular symmetry to the modal shapes except the mirror symmetry about the $x_{3}=0$ plane; hence, the mixing of in-plane shears and longitudinal strains is not anyhow constrained. The mode $\mathrm{F}-\mathrm{F}^{\prime}-\mathrm{F}^{\prime \prime}$ is dominantly shear for $k \lesssim 0.4(2 \pi / a)$ while the mode $\mathrm{G}-\mathrm{G}^{\prime}-\mathrm{G}^{\prime \prime}$ is dominantly longitudinal in this region (see visualizations F and G). However, when the curves for these two modes nearly touch each other at $k \sim 0.45(2 \pi / a)$, their polarizations interchange (see visualizations $\mathrm{F}^{\prime}$ and $\mathrm{G}^{\prime}$ ), which repeats at $k \sim 0.9(2 \pi / a)$, and so the mode $\mathrm{F}-\mathrm{F}^{\prime}-\mathrm{F}^{\prime \prime}$ is purely shear again at the edge of the Brillouin zone (visualization $\mathrm{F}^{\prime \prime}$ ), while the mode $\mathrm{G}-\mathrm{G}^{\prime}-\mathrm{G}^{\prime \prime}$ is purely longitudinal at this point. The lowest frequency 

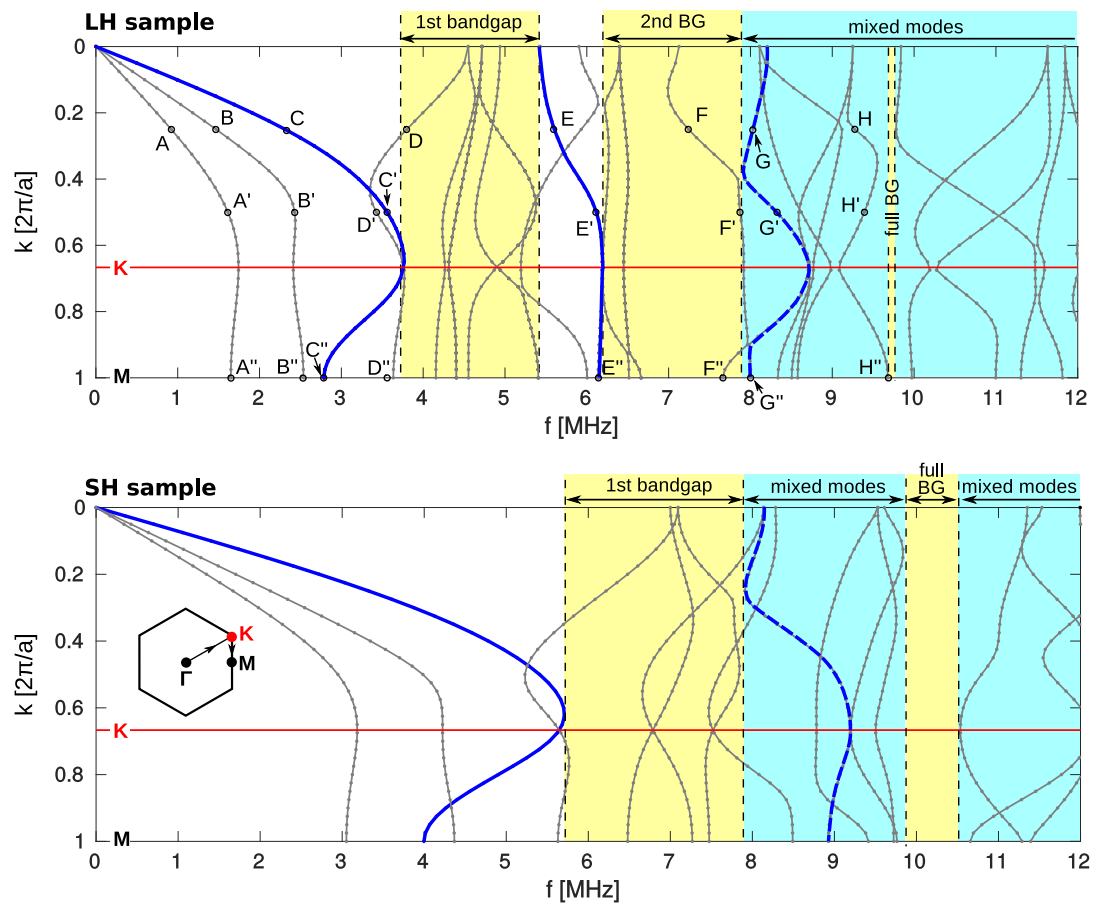

Figure 9: Band structure of the hexagonal scaffolds LH (upper plot) and SH (lower plot) calculated by FEM. The thick (blue) lines denote the modes with dominant longitudinal component, the thick dashed lines denote modes with mixed polarizations but having a dominant longitudinal component in some segments. The shaded region labeled as 'Mixed modes' covers the modes among which a distinguishing between dominantly longitudinal a dominantly shear ones is impossible. In the upper plot, the capital letters A, . ,., $\mathrm{H}^{\prime \prime}$ refer to Fig.10; within the lower plot area, the first Brillouin zone of the hexagonal environment is sketched, with marked points $\Gamma$ (center), $\mathrm{K}$ and $\mathrm{M}$.

of the longitudinal polarization of these two modes corresponds well to the end of the second experimentally obtained BG, and so it can be concluded that although the mode $\mathrm{G}-\mathrm{G}^{\prime}-\mathrm{G}^{\prime \prime}$ is of the above described mixed polarization, this mode enables some energy transmission through the structure.

All higher modes exhibit similar switching between longitudinal and shear polarizations, or even more complex modal shapes (such as $\mathrm{H}-\mathrm{H}^{\prime}-\mathrm{H}^{\prime \prime}$ ) in which the longitudinal and shear components are fully comparable. Hence, it is difficult to find the origin of the third experimental BG for the LH structure. The calculations predict a narrow full (total) BG at 9.7-9.8 MHz, but the relation of this calculated BG and the broad observed minimum of the transmitted amplitude is questionable.

Similarly, for the SH sample, the FEM calculation enables a straightforward interpretation only for the lowest modes. The start of the first BG again corresponds to the 
stationary point of the longitudinal acoustic mode. The end of this BG then probably corresponds to the mode denoted by the dashed line in the lower subplot of Fig.9. This mode is geometrically similar to the mode $G-G^{\prime}-G^{\prime \prime}$ of the $S H$ structure, and exhibits similar switching of polarizations. However, at higher frequencies the agreement between the model and experiment deteriorates. The FEM calculations predict a full BG between 9.8 and $10.6 \mathrm{MHz}$, which cannot be seen in the experimental results. On the contrary, the experiment gives a local maximum of the transmitted amplitude in this interval. It is plausible that this theoretically predicted full BG may correspond to the measured BG at above $11.0 \mathrm{MHz}$, and the discrepancy follows from the deviations of the real micromorphology of the scaffold from the geometry of the FEM model. Similarly as for the tetragonal scaffolds, the agreement between the model and the experiment at high frequencies is worse for the denser structure. As it can be partially seen from the optical micrographs in Fig.1, the rods in these dense structures (SS and $\mathrm{SH}$ ) appear more wavy, exhibiting less constant thickness over the structure; hence, a stronger disagreement with the model may be expected.

\section{Conclusions}

Both experimental results and FEM calculations in the present paper confirm that ceramic microlattices fabricated by Robocasting exhibit band structures for longitudinal ultrasonic wave propagation with acoustic bandgaps. For the particular studied fine robocast scaffolds, the lowest BGs appear above $3 \mathrm{MHz}$. Broad BGs were observed for both tetragonal and hexagonal structures, the spectral width of these gaps being between $\sim 1$ $\mathrm{MHz}$ and $\sim 2 \mathrm{MHz}$ in both cases. Robocasting, as a 3D printing method, is proved to be a versatile tool for rapid prototyping of phononic crystals with a prescribed acoustic band structure and bandgaps that block acoustic energy propagation at frequency ranges of as high as $5-10 \mathrm{MHz}$.

The results presented in this paper also show the limitations for FEM modeling of the wave propagation in real robocast structures. For increasing frequencies, the geometric imperfections and/or boundary defects of the real structures may lead to significant shifts between the predicted locations of the bandgaps and the real frequency ranges in which 


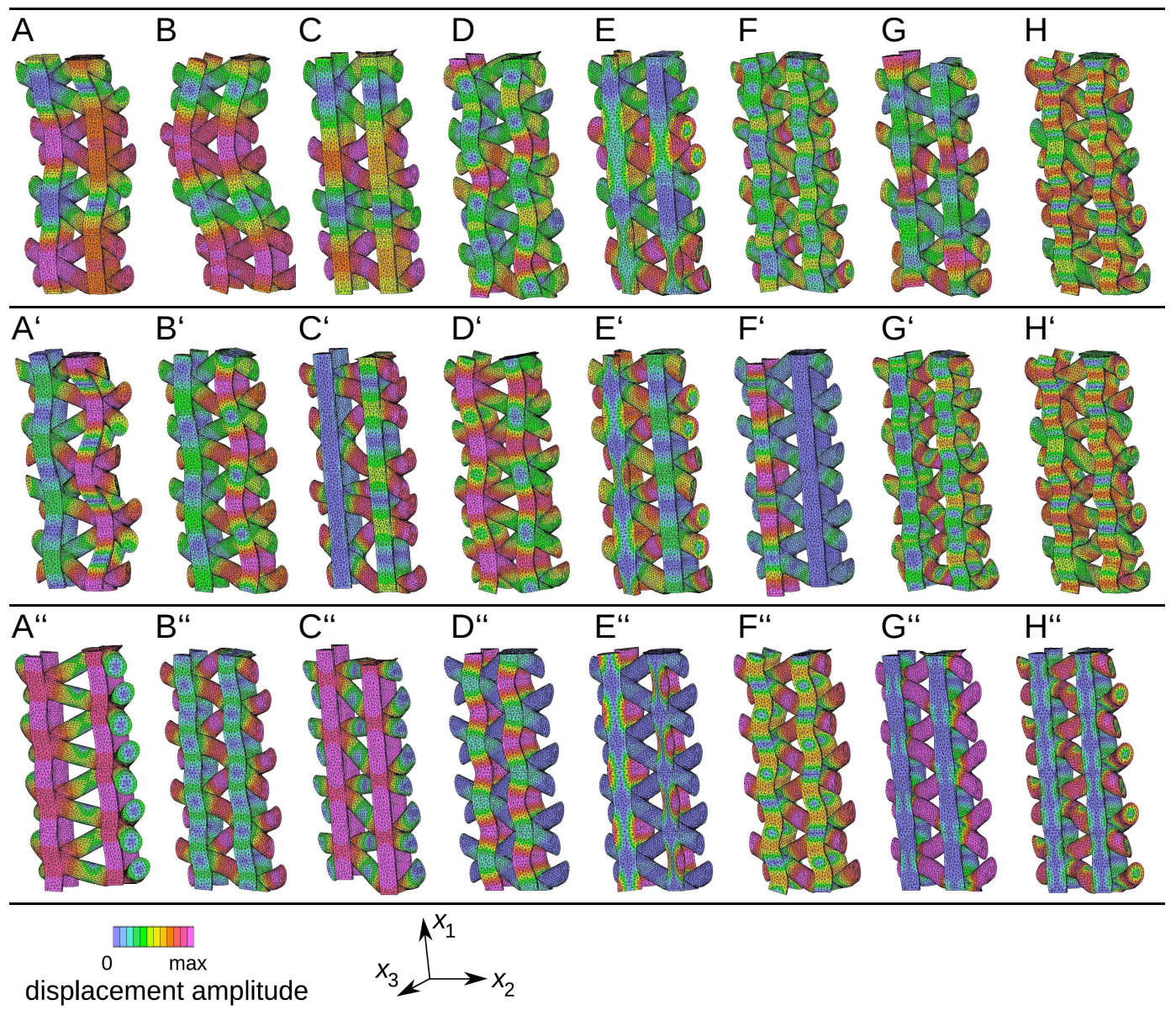

Figure 10: Visualizations of the selected propagation modes (see the labels in Fig. 9) for the LH structure. The color-scale represents the instantaneous displacement amplitude for fixed phase angle $\omega t$.

the transmitted energy is lowered. Despite this quantitative mismatch at high frequencies, FEM calculations were shown efficient for the interpretation of the experimentally observed band structures, and enabled also a deeper insight into the acoustic wave propagation in the scaffolds, especially for the hexagonal symmetry class where mixing of shear and longitudinal polarizations appears.

\section{Acknowledgment}

This work was financially supported by the Czech Science Foundation (grant No.1701618S) and the Spanish Government under project MAT2015-67437-R. 


\section{References}

[1] J. E. Smay, G. Gratson, R. Shepherd, J. Cesarano, J. A. Lewis, Directed Colloidal Assembly of 3D Periodic Structures, Adv. Mater. 14 (2002) 1279-1283.

[2] J.E. Smay, J.A. Lewis, Solid Free-Form Fabrication of 3-D Ceramic Structures, in: N.P. Bansal, A.R. Boccaccini (Eds.), Ceramics and Composites Processing Methods, John Wiley and Sons, Hoboken, 2012, pp. 459-484.

[3] K. Cai, B. Román-Manso, J.E. Smay, J. Zhou, M.I. Osendi, M. Belmonte, P. Miranzo, Geometrically Complex Silicon Carbide Structures Fabricated by Robocasting, J. Am. Ceram. Soc. 95 (2012) 2660-2666.

[4] P. Miranda, E. Saiz, K. Gryn, A.P. Tomsia, Sintering and robocasting of $\beta$-tricalcium phosphate scaffolds for orthopaedic applications, Acta Biomater. 2 (2006) 457-466.

[5] D. L. Cohen, J. I. Lipton, L. J. Bonassar, H. Lipson, Additive manufacturing for in situ repair of osteochondral defects, Biofabrication 2 (2010) 035004.

[6] B. Román-Manso, S.M. Vega-Díaz, A. Morelos-Goméz, M. Terrones, P. Miranzo, M. Belmonte, Aligned carbon nanotube/silicon carbide hybrid materials with high electrical conductivity, superhydrophobicity and superoleophilicity, Carbon 80 (2014) 120-126.

[7] A. Kruisová, H. Seiner, P. Sedlák, M. Landa, B. Román-Manso, P. Miranzo, M. Belmonte, Metamaterial behavior of three-dimensional periodic architectures assembled by robocasting, Appl. Phys. Letters 105 (2014) 211904.

[8] R.V. Craster, S. Guenneau (Eds.) Acoustic Metamaterials: Negative Refraction, Imaging, Lensing and Cloaking, Springer Series in Materials Science Vol. 166, Springer, London, 2013.

[9] S. Guenneau, A. Movchan, G. Petursson, S. A. Ramakrishna, Acoustic metamaterials for sound focusing and confinement, New J. Phys. 9 (2007) 399. 
[10] J.-H. Lee, C. Y. Koh, J. P. Singer, S.-J. Jeon, M. Maldovan, O. Stein, E. L. Thomas, 25th Anniversary Article: Ordered Polymer Structures for the Engineering of Photons and Phonons, Adv. Mater. 26 (2014) 532-569.

[11] T. Gorishnyy, M. Maldovan, C. Ullal, and E. L. Thomas, Sound Ideas, Phys. World 18 (2005) 24-29.

[12] S. Yang, J. H. Page, Z. Liu, M. L. Cowan, C. T. Chan, P. Sheng, Focusing of Sound in a 3D Phononic Crystal, Phys. Rev. Lett. 93 (2004) 024301.

[13] X. Zhang, Z. Liu, Negative refraction of acoustic waves in two-dimensional phononic crystals, Appl. Phys. Lett. 85 (2004) 341-343.

[14] H. Zhao, Y. Liu, D. Yu, G. Wang, J. Wen, X. Wen, X, Absorptive properties of three-dimensional phononic crystal, J. Sound Vib. 303 (2007) 185-194.

[15] A. Kruisová, H. Seiner, P. Sedlák, M. Landa, B. Román-Manso, P. Miranzo, M. Belmonte, Finite elements modeling of mechanical and acoustic properties of a ceramic metamaterial assembled by robocasting, Appl. Mech. Mater. 821 (2016) 364-371.

[16] Y. Liu, J.-Y. Su, Y.-L. Xu, X.-C. Zhang, The influence of pore shapes on the band structures in phononic crystals with periodic distributed void pores, Ultrasonics 49 (2009) 276-280.

[17] Y. Liu, J.-Y. Su, L. Gao, The influence of the micro-topology on the phononic bandgaps in 2D porous phononic crystals, Phys. Lett. A 372 (2008) 6784-6789.

[18] Z. Hou, Y. Liu, The effects of shapes and symmetries of scatterers on the phononic band gap in 2D phononic crystals, Phys. Lett. A 332 (2004) 481-490.

[19] M. Miniaci, A. Marzani, N. Testoni, L. D. Marchi, Complete bandgaps in a polyvinyl chloride (pvc) phononic plate with cross-like holes: numerical design and experimental verification, Ultrasonics, 56 (2015) 251-259.

[20] P. Celli, S. Gonella, Laser-enabled experimental wavefield reconstruction in twodimensional phononic crystals, J. Sound. Vib. 333 (2014) 114-123. 
[21] O. Yuksel, C. Yilmaz, Shape optimization of phononic band gap structures incorporating inertial amplification mechanisms, J. Sound. Vib. 355 (2015) 232-245.

[22] J. O. Vasseur, P. A. Deymier, B. Chenni, B. Djafari-Rouhani, L. Dobrzynski, D. Prevost, Experimental and Theoretical Evidence for the Existence of Absolute Acoustic Band Gaps in Two-Dimensional Solid Phononic Crystals, Phys. Rev. Lett. 86 (2001) 3012-3015

[23] M. Torres, F.R. Montero de Espinosa, Ultrasonic bandgaps and negative refraction, Ultrasonics 42 (2004) 787-790

[24] B. Román-Manso, Á. de Pablos, M. Belmonte, M. I. Osendi, P. Miranzo, Microstructural designs of spark-plasma sintered silicon carbide ceramic scaffolds, Bol. Soc. Esp. Ceram. Vidr. 53 (2014) 93-100.

[25] Comsol Inc., Comsol Multiphysics version 3.5, (2007).

[26] Y. Liu, X. Z. Sun, S. T. Chen, Band gap structures in two-dimensional super porous phononic crystals, Ultrasonics, 53 (2013) 518-524.

[27] Y.-F. Wang, Y.-S. Wang, X.-X. Su, Large bandgaps of two-dimensional phononic crystals with cross-like holes, J. Appl. Phys. 110 (2011) 113520.

[28] F. Bloch, Quantum mechanics of electrons in crystal lattices, Z. Phys. 53 (1928) 555-600.

[29] C. Kittel, Introduction to the Solid State Physics, eighth ed., Wiley, New York, 2005. 